\title{
Jardín botánico y arboretum: estrategias de conservación forestal en paisajes antropizados del trópico mexicano
}

\author{
Botanical garden and arboretum: forest conservation \\ strategies in anthropized landscapes of the Mexican tropics
}

\author{
Ligia Guadalupe Esparza-Olguín ${ }^{1 *}$, Aixchel Maya Martínez², Gonzalo Hernández García²
} y Eduardo Martínez Romero ${ }^{3}$

\begin{abstract}
Botanical gardens and arboreta are considered relevant conservation instruments based on the alarming deforestation rates that forests have and the consequent impact on their diversity. In this paper, the diversity, conservation status and potential use of tree species in the botanical garden (JB) and arboretum (A) of the El Tormento Forest Technology Research and Transfer Center were analyzed. In the first, the sampling was carried out in a total area of $9375 \mathrm{~m}^{2}$; while in the second the sampling was done in 1.6 ha. Both spaces represent vegetation of medium sub-evergreen forest, with 11871 individuals which belong to 92 species and 35 families. JB was 1.6 times more diverse than A. 15 potential uses were documented in $98.9 \%$ of the species with at least one registered use. Among the species in both collections, there are seven classified in the IUCN red list, two in CITES and eight in the NOM-059-SEMARNAT-2010. These results underscore the importance of the botanical garden and the arboretum as reservoirs of useful forest species from many different perspectives and as conservation spaces for threatened taxa from the medium-long sub-evergreen forests of southern Mexico.
\end{abstract}

Key words: El Tormento Forest Technology Research and Transfer Center, deforestation, tree species, conservation status, forest management, semi evergreen tropical forest.

\begin{abstract}
Resumen
Los jardines botánicos y arboreta son considerados instrumentos de conservación relevantes a partir de las alarmantes tasas de deforestación que tienen las selvas y la consecuente afectación de su diversidad. En este trabajo se analizó la diversidad, el estatus de conservación y el uso potencial de las especies arbóreas en el jardín botánico (JB) y el arboretum (A) del Centro de Investigación y Transferencia de Tecnología Forestal El Tormento. En el primero, el muestreo se realizó en un área total de $9375 \mathrm{~m}^{2}$; mientras que, en el segundo el muestreo se hizo en 1.6 ha. Ambos espacios representan vegetación de selva mediana subperennifolia, con 11871 individuos pertenecientes a 92 especies y 35 familias. El JB fue 1.6 veces más diverso que A. Se documentaron 15 usos potenciales en $98.9 \%$ de los taxones con al menos un uso registrado. Entre los taxa de ambas colecciones, se tienen siete clasificadas en la lista roja de la IUCN, dos en CITES y ocho en la NOM-059SEMARNAT-2010. Estos resultados subrayan la importancia del jardín botánico y el arboretum como reservorios de especies forestales útiles desde muy diversas perspectivas y como espacios de conservación de taxa amenazados de las selvas medianas subperennifolias del sur de México.
\end{abstract}

Palabras clave: Centro de Investigación y Transferencia de Tecnología Forestal El Tormento, deforestación, especies arbóreas, estatus de conservación, manejo forestal, selva subperennifolia.

Fecha de recepción/Reception date: 31 de enero de 2020

Fecha de aceptación/Acceptance date: 19 de junio de 2020

${ }^{1}$ Departamento de Ciencias de la Sustentabilidad, El Colegio de la Frontera Sur, Unidad Campeche. México

${ }^{2}$ Centro Experimental Edzná, CIR-Sureste, INIFAP. México

${ }^{3}$ Investigación y Soluciones Socioambientales, A.C. Campeche, México

*Autor por correspondencia; correo-e: Igeo.ecosur@gmail.com 


\section{Introducción}

Las selvas mantienen más de la mitad de las especies del planeta y se consideran importantes centros de endemismos (Dirzo et al., 2009). Son reguladoras del ciclo hidrológico y de la temperatura ambiental (Lawrence et al., 2004); además de que proporcionan diversos recursos a las comunidades humanas como alimento, madera, vestido, medicina, recreación, entre otros (Balvanera, 2012). Estos ecosistemas se cuentan entre los más afectados por actividades antropogénicas de tipo urbano, agrícola y ganadero; además por la incidencia de fenómenos naturales como huracanes e incendios forestales. Lo anterior ha provocado la pérdida (deforestación), degradación o fragmentación de una gran parte de las selvas (FAO, 2016); lo que ha conducido a la pérdida de biodiversidad y de una gran cantidad de recursos forestales potencialmente útiles para el hombre (Sloan y Soyer, 2015).

En México, se reconoce que la deforestación ha tenido un mayor impacto en las selvas que en los bosques templados, misma que se estima en una reducción de $80 \%$ de su territorio en el país (Challenger y Soberón, 2008). Los procesos de deforestación y fragmentación han sido muy importantes en las selvas del sur de la Península de Yucatán, que albergan una riqueza florística calculada en 161 familias que agrupan a 2329 especies, de las cuales $8.6 \%$ son endémicas de la región (Pérez-Sarabia et al., 2017).

Una estrategia para salvaguardar los recursos forestales son los jardines botánicos y los arboreta, como centros de conservación ex-situ o in-situ, pues desempeñan una función muy importante en programas de educación ambiental, investigación y entrenamiento en el conocimiento botánico a diversos niveles (Arnet et al., 2015; Dunn, 2017; Chen y Sun, 2018). Son particularmente valiosos cuando preservan áreas de bosques o selvas inmersos en matrices agropecuarias o urbanas, pues constituyen hábitats o corredores que coadyuvan en la conservación de la flora y la fauna (Dunn, 2017). 
El jardín botánico (JB) del Centro de Investigación y Transferencia de Tecnología Forestal "El Tormento", ubicado en Campeche, México, fue fundado en 1965 como un jardín natural forestal representativo de la selva mediana subperennifolia, con el objetivo de preservar la diversidad forestal de este ecosistema e introducir especies del trópico mexicano o de otras partes del mundo para enriquecerlo (SalayaSánchez y Gómez-Gómez, 1981). El arboretum (A), se estableció en 1963 con la finalidad de representar una plantación forestal comercial mixta de especies nativas y exóticas, así como para conservar parte de la diversidad genética de especies forestales maderables, con fines de investigación y educación (Uzcanga-Pérez et al., 2018). Desafortunadamente, ambas áreas fueron abandonadas por diferentes razones durante más de veinte años. En la actualidad, tanto el JB como el A, están en proceso de reactivación, con el propósito de constituir un espacio para actividades de educación ambiental, investigación científica, así como reserva de especies nativas y exóticas con usos múltiples.

El objetivo del presente estudio consistió en evaluar el estado de conservación actual de la vegetación del jardín botánico y del arboretum del Centro de Investigación y Transferencia de Tecnología Forestal El Tormento, por medio del análisis de la representatividad de las especies arbóreas y la estructura en ambos espacios, respecto a las comunidades vegetales circundantes, la diversidad de especies arbóreas en ambos espacios, y su uso potencial. Esta información documentará las estrategias de reactivación en dichas colecciones vivas y contribuirá en los esfuerzos de conservación de las selvas.

\section{Materiales y Métodos}

El Centro de Investigación y Transferencia de Tecnología Forestal El Tormento se encuentra a $7 \mathrm{~km}$ de la ciudad de Escárcega, en el kilómetro 292 de la carretera federal 186, entre los $18^{\circ} 16^{\prime} 25^{\prime \prime} \mathrm{N}$ y los 9043'55" O en el municipio Escárcega, Campeche (Cedeño, 1981). El clima en la región es tropical, $A(w)$ l'g, según la 
clasificación de Köppen, modificada por García, con temperaturas medias anuales entre 23 y $25{ }^{\circ} \mathrm{C}$, con máximas de $42{ }^{\circ} \mathrm{C}$ y mínimas de $4.5{ }^{\circ} \mathrm{C}$; con una precipitación media anual de 1145 mm (López-Torres y Tamarit-Urías, 2005).

EI JB se ubica en el sureste del Centro de Investigación, a un costado de la zona habitacional; mientras que el A en la entrada principal a las oficinas, ambos frente a la carretera 186 (Villahermosa-Escárcega). El JB ocupa un área de $800 \mathrm{~m}$ de largo y $50 \mathrm{~m}$ de ancho (4 ha), mientras que el A tiene una superficie de 1.6 ha y está dividido en dos cuadrantes, separados por un camino de acceso a las oficinas, establecidos como una colección de árboles vivos (Figura 1). En ambos casos los suelos predominantes son Rendzina y Vertisol, según el sistema de clasificación de FAO (Cuanalo, 1981).

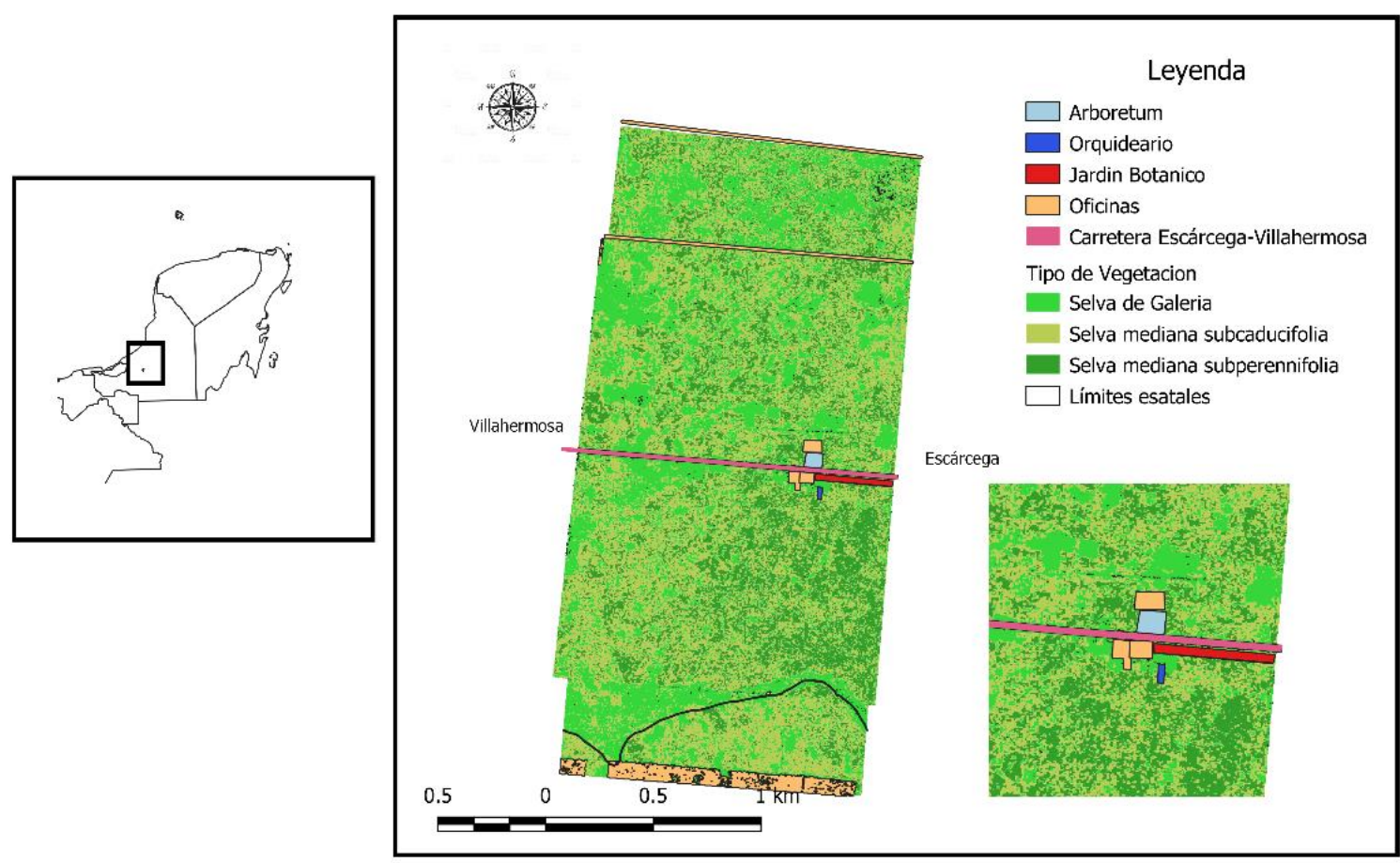

Figura 1. Ubicación del Centro de Investigación y Transferencia de Tecnología Forestal "El Tormento", el jardín botánico (JB) y el arboretum (A). 
El muestreo en campo se realizó en 15 parcelas cuadradas de $25 \times 25 \mathrm{~m}\left(625 \mathrm{~m}^{2}\right)$; es decir, en $9375 \mathrm{~m}^{2}$ en el JB; en el A se trabajó en los dos cuadrantes del área total y en las selvas circundantes (SM) se establecieron cinco parcelas circulares de $1000 \mathrm{~m}^{2}$ (17.84 $\mathrm{m}$ de diámetro). En estas áreas se identificó a los individuos arbóreos presentes hasta nivel de especie, a los que se les midió el diámetro a la altura de $1.30 \mathrm{~m}$ a partir de $2.5 \mathrm{~cm}$, con una cinta diamétrica modelo 283D/5m Forestry Suppliers, y la altura con un hipsómetro Vertex IV Hanglöf.

La identificación taxonómica se realizó con el apoyo de parataxónomos (Demetrio Álvarez Montejo, Manuel Arana Cua y Antonio López Carrillo), bibliografía especializada (Pennington y Sarukhán, 2005; Carnevali et al., 2010) y el apoyo de expertos del herbario de la Universidad Autónoma de Campeche. Para la corroboración y, en su caso, la actualización de los datos taxonómicos se consultó The plant list (2013).

Con los datos de campo se hizo un listado florístico de las especies arbóreas, cuyo uso se documentó mediante referencias especializadas y publicadas, así como su estatus de conservación en la NOM-059-SEMARNAT-2010 (Semarnat, 2010), la lista roja de la Unión Internacional para la Conservación de la Naturaleza (IUCN, 2019) y los apéndices de la Convención sobre el Comercio Internacional de Especies Amenazadas de Fauna y Flora Silvestre (CITES, 2017).

Para examinar la representatividad de los taxones del JB y A respecto a las SM, se compararon los listados, se analizó la diferencia en la composición de especies entre JB y SM, así como A y SM mediante un análisis de similitud (ANOSIM), para ello se utilizó la distancia del índice de Bray Curtis (Bray y Curtis, 1957). La estimación de la proporción que aporta cada taxón a la diferencia entre las áreas de estudio, se determinó por el porcentaje de disimilitud entre ellas, se empleó la subrutina SIMPER (Clarke, 1993). Estos análisis se hicieron con el programa PAST 3.25 (Hammer et al., 2001). 
Se calcularon la densidad (ind $\left.\mathrm{ha}^{-1}\right)$, el área basal $\left(\mathrm{m}^{2} \mathrm{ha}^{-1}\right)$ y el valor de importancia relativa de las especies (VIR). El VIR correspondió a la sumatoria de la abundancia relativa (número de individuos por especies/número total de individuos de todas las especies * 100); la frecuencia relativa (frecuencia de una especie/suma de la frecuencia de todas las especies * 100); y área basal relativa (área basal de cada especie/área basal total de todas las especies * 100) (Magurran, 2004).

En el análisis de la estructura horizontal se emplearon los valores de diámetro a la altura del pecho y para la vertical los datos de la altura total, que se representaron mediante distribuciones de frecuencia agrupadas por clase en histogramas.

Se estimaron para el JB y A la riqueza de familias (número de familias) y la diversidad verdadera mediante la expresión:

$$
q D=\left(\sum_{i}^{S}=1 p_{i}^{q}\right)^{1 /(1-q)}
$$

Donde:

$q D=$ Diversidad verdadera

$p_{i}=$ Abundancia relativa (abundancia proporcional) de la iésima especie

$S=$ Número de especies

$q=$ Orden de la diversidad

Esta expresión define la sensibilidad del índice a las abundancias relativas de las especies (Jost y González-Oreja, 2012). Las medidas de diversidad verdadera estimadas fueron las de orden cero (OD), es decir la riqueza específica, y la de orden 1 (1D) que considera a todas las especies en el valor de diversidad. Además, al estimar la riqueza esperada para las áreas de estudio se calculó la eficiencia de 
muestreo o completitud del inventario, mediante la comparación de los taxones observados y estimados; para ello, se emplearon los estimadores no paramétricos Chao1, ACE (basados en la incidencia de las especies) y Chao 2 (basado en la abundancia de individuos de las especies) con el software Estimates versión 9.1 (Colwell, 2017).

\section{Resultados}

Se registraron 11871 individuos: 10723 en el JB (11 457 ind ha-1) y 1148 en el A (717.5 ind ha-1). Se documentaron 92 especies pertenecientes a 35 familias, de las cuales 86 se encontraron en el JB y 51 en el A; de ellas, las mejor representadas por el número de especies en los dos sitios fueron Fabaceae (19), Arecaceae (7), Sapotaceae (6) y Rubiaceae (5), que incluyen $40 \%$ de los taxones en ambas áreas de estudio (Cuadro 1). Las familias con mayor número de individuos fueron Sapotaceae (1 369), Arecaceae, Anacardiaceae, Rubiaceae, Lauraceae y Fabaceae (Cuadro 1).

Cuadro 1. Abundancia y usos de las especies arbóreas registradas en el jardín botánico (JB) y en el arboretum (A) El Tormento.

\begin{tabular}{|c|c|c|c|}
\hline Familia/Especie & A & JB & Usos \\
\hline \multicolumn{4}{|l|}{ Anacardiaceae } \\
\hline Astronium graveolens Jacq. & 65 & 231 & M \\
\hline Metopium brownei (Jacq.) Urb. & 26 & 672 & $\mathrm{Me}, \mathrm{C}$ \\
\hline Spondias mombin L. & 63 & 29 & $\mathrm{~A}, \mathrm{M}, \mathrm{C}, \mathrm{Md}, \mathrm{CV}, \mathrm{U}, \mathrm{Pa}$ \\
\hline \multicolumn{4}{|l|}{ Annonaceae } \\
\hline Annona reticulata $\mathrm{L}$. & 1 & 71 & $\mathrm{~A}, \mathrm{Me}, \mathrm{Md}$ \\
\hline Mosannona depressa (Baill.) Chatrou & & 24 & $\mathrm{Me}, \mathrm{M}, \mathrm{Cr}$ \\
\hline \multicolumn{4}{|l|}{ Apocynaceae } \\
\hline Thevetia ahouai (L.) A.DC. & & 4 & $\mathrm{M}, \mathrm{Me}, \mathrm{O}$ \\
\hline \multicolumn{4}{|l|}{ Araliaceae } \\
\hline Dendropanax arboreus (L.) Decne. \& Planch & 4 & 167 & $\mathrm{Md}, \mathrm{M}, \mathrm{Ar}, \mathrm{C}, \mathrm{Me}, \mathrm{Pa}$ \\
\hline Schefflera morototoni (Aubl.) Maguire, Steyerm. \& Frodin & 1 & 2 & $\mathrm{Md}, \mathrm{M}, \mathrm{I}$ \\
\hline \multicolumn{4}{|l|}{ Arecaceae } \\
\hline Chamaedorea oblongata Mart. & & 130 & 0 \\
\hline
\end{tabular}


Chamaedorea seifrizii Burret

Cryosophila stauracantha (Heynh.) R.J.Evans

Desmoncus orthacanthos Mart.

Gaussia maya (O.F. Cook) H.J.Quero \& Read

Sabal gretherae H. J. Quero

Sabal mexicana Mart.

Asparagaceae

Beaucarnea pliabilis (Baker) Rose

Bignoniaceae

Handroanthus chrysanthus (Jacq.) S.O.Grose

Tabebuia rosea (Bertol.) Bertero ex ADC.

Tecoma stans (L.) Juss. ex Kunth

Boraginaceae

Cordia dodecandra A. DC.

Ehretia tinifolia L.

Burseraceae

Bursera simaruba (L.) Sarg.

60

Protium copal (Schltdl. \& Cham.) Engl.

Cannabaceae

Aphananthe monoica (Hemsl.) J.F.Leroy

Clusiaceae

Calophyllum brasiliense Cambess.

Combretaceae

Bucida buceras L.

Erythroxylaceae

Erythroxylum confusum Britton

Euphorbiaceae

Alchornea latifolia Sw.

Gymnanthes lucida Sw.

Hippomane mancinella L.

Fabaceae

Acacia cornígera (L.) Willd

Acosmium panamense (Benth.) Yakovlev

Albizia niopoides (Benth.) Burkart

Bauhinia divaricata L.

Delonix regia (Hook.) Raf.

Enterolobium cyclocarpum (Jacq.) Griseb.

Gliricidia maculata ("Humb., Bonpl. \& Kunth") Steud.

Lonchocarpus castilloi Standl.

Lonchocarpus guatemalensis Benth.

$560 \quad \mathrm{O}, \mathrm{Ar}$

\section{5}

1

4

4

3

131

$\mathrm{U}, \mathrm{M}, \mathrm{O}$

$\mathrm{Ar}$

$\mathrm{O}$

O

$\mathrm{O}, \mathrm{Ar}, \mathrm{Md}$

20

\begin{tabular}{ccc} 
& 3 & Md, O, CV, U, Me, M, C \\
18 & 1 & A, C, M, Md, Me, U \\
& 87 & C, Md, CV , M, O, E, F, I, Me \\
\hline
\end{tabular}

8 9

247

44
$\mathrm{Md}, \mathrm{A}, \mathrm{U}, \mathrm{M}, \mathrm{Ar}$ $\mathrm{U}, \mathrm{M}, \mathrm{O}, \mathrm{Md}, \mathrm{Me}, \mathrm{Ar}$ 
Lonchocarpus longistylus Pittier

Lotus berthelotii Masf.

Lysiloma latisiliquum (L.) Benth.

Mariosousa dolichostachya (S.F. Blake) Seigler \& Ebinger

Myroxylon balsamum (L.) Harms

Piscidia piscipula (L.) Sarg.

Platymiscium yucatanum Standl.

Schizolobium parahyba (Vell.) S.F. Blake

Swartzia cubensis (Britton \& Wilson) Standl.

Vatairea lundellii (Standl.) Record

Lamiaceae

Gmelina arborea Roxb.

Tectona grandis L. f.

Vitex gaumeri Greenm.

Lauraceae

Licaria peckii (I.M.Johnst.) Kosterm.

Nectandra salicifolia (Kunth) Nees.

Malvaceae

Guazuma ulmifolia Lam.

Hampea trilobata Standl.

Pseudobombax ellipticum (Kunth) Dugand

Meliaceae

Cedrela odorata L.

Swietenia macrophylla King

Moraceae

Brosimum alicastrum Sw.

Pseudolmedia glabrata (Liebm.) C.C.Berg

Trophis racemosa (L.) Urb.

Myrtaceae

Eucalyptus $s p$

Pimenta dioica (L.) Merrill

Psidium sartorianum (O.Berg) Nied.

Phyllanthaceae

Margaritaria nobilis L.f.

Picramniaceae

Alvaradoa amorphoides Liebm.

7

Piperaceae

Piper aduncum L.

Polygonaceae

Coccoloba barbadensis Jacq.
1

2

77

\begin{tabular}{|c|c|c|}
\hline & 2 & $\mathrm{CR}, \mathrm{O}, \mathrm{M}$ \\
\hline & 8 & $\mathrm{O}$ \\
\hline 131 & 177 & $\mathrm{Md}, \mathrm{Me}, \mathrm{C}, \mathrm{Cn}, \mathrm{F}, \mathrm{M}, \mathrm{U}$ \\
\hline 31 & 259 & $\mathrm{Md}, \mathrm{Me}$ \\
\hline 2 & 3 & $\mathrm{I}, \mathrm{Md}, \mathrm{M}, \mathrm{Me}, \mathrm{E}$ \\
\hline 176 & 26 & $\mathrm{M}, \mathrm{Md}, \mathrm{Me}, \mathrm{C}, \mathrm{CV}, \mathrm{F}$ \\
\hline & 1 & $\mathrm{Md}, \mathrm{U}, \mathrm{Ar}$ \\
\hline & 4 & $\mathrm{Ar}, \mathrm{C}, \mathrm{Md}, \mathrm{Pa}$ \\
\hline 1 & 17 & $\mathrm{Me}, \mathrm{C}, \mathrm{M}, \mathrm{Md}$ \\
\hline & 5 & Md \\
\hline
\end{tabular}

78

1107

11

85

12

77

31

3

5

2

3

34
125

127

307

Md, C, M

$\mathrm{Md}$

$C, M, C V, M e, A r, U$
$\mathrm{C}, \mathrm{CV}, \mathrm{Me}$
Md 
Revista Mexicana de Ciencias Forestales Vol. 11 (60)

Julio - Agosto (2020)

Coccoloba cozumelensis Hemsl.

2

196

$\mathrm{Md}, \mathrm{Me}$,

Putranjivaceae

Drypetes lateriflora (Sw.) Krug \& Urb

2

153

$M d, C R$

Rhamnaceae

Krugiodendron ferreum (Vahl) Urb

1

11

Md

Rosaceae

Crataegus rhipidophylla Gand.

34

$\mathrm{O}$

\section{Rubiaceae}

Alseis yucatanensis Standl.

23

479

Md, Me

Blepharidium guatemalense Standl.

$20 \quad 72$

Exostema mexicanum A. Gray

$5 \quad 225$

Guettarda combsii Urb

$2 \quad 287$

Simira salvadorensis (Standl.) Steyerm

1

287

Md, C, Me

Md

Rutaceae

Zanthoxylum caribaeum Lam.

1

34

$\mathrm{U}, \mathrm{Md}$

Salicaceae

Laetia thamnia L.

116

M

Zuelania guidonia (Sw.) Britton \& Millsp.

$3 \quad 105$

$M, C R, M d$

$M, M d, C$

Sapindaceae

Cupania glabra Sw.

430

Md

Matayba oppositifolia (A.Rich.) Britton \& Millsp.

9

$\mathrm{Md}$

Melicoccus oliviformis subsp. intermedius (Radkl.) Acev. Rodr

13

A, C, Md

Sapotaceae

Chrysophyllum mexicanum Brandegee

\section{4}

Manilkara zapota L(L.) P.Royen

3163

Pouteria campechiana (Kunth) Baehni

1

Pouteria reticulata (Engl.) Eyma

Sideroxylon foetidissimum Jacq.

Sideroxylon salicifolium (L.) Lam.

Simaroubaceae

Simarouba amara Aubl.

33

134

130

946

5

96

A, Md, M

$A, M d, M, I, A r, F, U$

Md, A, M, Me

$M d, A$

Md, F, M, Me, I

$\mathrm{M}, \mathrm{Md}, \mathrm{Me}, \mathrm{A}$

Urticaceae

Cecropia peltata L.

2

$M, A r, A, I, F, U, P a, M d$

Usos: $A=$ Alimento; $M=$ Medicina tradicional $C=$ Combustible; $M d=$ Madera;

$\mathrm{O}=$ Ornato; $\mathrm{Ar}=$ Artesanías $; \mathrm{U}=$ Utensilios; $\mathrm{P}=$ Pegamento $; \mathrm{CV}=$ Cercos

vivos; $F=$ Forraje; $C R=$ Cultural, religioso; $E=$ Esencias, cosméticos, jabones;

$\mathrm{Me}=$ Melíferas; I = Etanol, insecticida, otros metabolitos secundarios usados en la industria; $\mathrm{Pa}=$ Papel. 
En relación con las especies, las más abundantes en el JB fueron Cryosophila stauracantha (Heynh.) R.J.Evans (1 145), Nectandra salicifolia (Kunth) Nees. (1 111), Pouteria reticulata (Engl.) Eyma (946), Metopium brownei (Jacq.) Urb. (672), y Chamaedorea seifrizii Burret (560); mientras que en el A fueron Piscidia piscipula (L.) Sarg. (176), Lysiloma latisiliquum (L.) Benth. (131), Lonchocarpus castilloi Standl. (89), Vitex gaumeri Greenm. y Cedrela odorata L. (77) (Cuadro 1).

Entre los taxa identificados en el JB y el A, se registraron siete especies endémicas; dos en ambas áreas de estudio: Mariosousa dolichostachya (S.F. Blake) Seigler \& Ebinger y Alseis yucatanensis Standl.; y cinco presentes solo en el JB: $C$. stauracantha, Gaussia maya (O.F. Cook) H.J. Quero \& Read, Lonchocarpus longistylus Pittier, Platymiscium yucatanum Standl. y Hampea trilobata Standl. Además, se registraron cinco especies introducidas, una compartida en ambas áreas: Myroxylon balsamum (L.) Harms (originaria de Centroamérica), una en el JB Lotus berthelotii Masf. (endémica de Tenerife) y tres en el A; Delonix regia (Hook.) Raf. (endémica de Madagascar), Gmelina arborea Roxb. (nativa del sudeste de Asia y la India) y Eucalyptus sp. (originaria de Australia y Nueva Guinea).

Respecto al estatus de conservación de los taxones registrados en el JB y A: siete especies están en la lista roja de IUCN, en las categorías en peligro (EN: Blepharidium guatemalense Standl.), vulnerable (VU: C. odorata y Swietenia macrophylla King), casi amenazada (NT: Mariosousa dolichostachya) y preocupación menor [LC: Tabebuia rosea (Bertol.) Bertero ex ADC., Lonchocarpus guatemalensis Benth. y Pouteria campechiana (Kunth) Baehni]. En CITES están dos especies en los apéndices II (S. macrophylla) y III (C. odorata); mientras que en la NOM-059SEMARNAT-2010 se registran ocho especies en las categorías en peligro de extinción [P: Vatairea lundellii (Standl.) Record], amenazada [A: Astronium graveolens Jacq., G. maya, Beaucarnea pliabilis (Baker) Rose, Handroanthus chrysanthus (Jacq.) S.O. Grose, Calophyllum brasiliense Cambess. y Acosmium panamense (Benth.) Yakovlev] y sujeta a protección especial (Pr: C. odorata). 
Por otro lado, en cuanto a la representación en el JB y el A del potencial de utilidad de las especies forestales tropicales, se identificaron 15 usos potenciales. Solamente uno de los taxones carece de citas de uso (B. guatemalense); mientras que $77.6 \%$ son especies multiuso (Cuadro 1). Entre los usos destacan el maderable, con $74.5 \%$ (madera para construcción, carpintería o ebanistería); medicinal (52.1\%); melífero, con $36.2 \%$ (provisión de néctar y polen); y combustible, con 34 \% (leña y carbón).

Se identificaron 103 especies en las SM, de las cuales 54 (52.43\%) estuvieron presentes en el JB y 38 en el A (36.89\%). El análisis ANOSIM mostró que la composición de especies en ambos casos fue estadísticamente diferente $(\mathrm{R}=0.769, \mathrm{p}=0.0086)$. EI análisis SIMPER permitió identificar una disimilitud promedio en este concepto de $83.96 \%$ entre las SM y el JB; y de $89.91 \%$ entre las SM y el $A$. Las especies que contribuyeron en mayor medida a esas diferencias se presentan en el Cuadro 2. 
Cuadro 2. Especies que contribuyen con los mayores porcentajes a la disimilitud en la composición de especies entre las SM y el JB, las SM y el A en El Tormento. Resultados obtenidos con el análisis SIMPER.

\begin{tabular}{|c|c|c|c|}
\hline Especie & $\mathrm{Pd}$ & $\%$ Contr. & $\%$ Contr. A \\
\hline Thouinia paucidentata Radlk. & 20.06 & 23.89 & 23.89 \\
\hline Cryosophila stauracantha (Heynh.) R. J.Evans & 5.892 & 7.018 & 30.91 \\
\hline Nectandra salicifolia (Kunth) Nees & 5.222 & 6.219 & 37.13 \\
\hline Pouteria reticulata (Engl.) Eyma & 4.74 & 5.646 & 42.78 \\
\hline Metopium brownei (Jacq.) Urb. & 3.545 & 4.222 & 47 \\
\hline Guettarda combsii Urb & 2.247 & 2.676 & 49.67 \\
\hline Cupania glabra Sw. & 2.162 & 2.575 & 52.25 \\
\hline Alseis yucatanensis Standl. & 2.119 & 2.524 & 54.77 \\
\hline Chamaedorea seifrizii Burret & 2.1 & 2.501 & 57.27 \\
\hline \multicolumn{4}{|l|}{ A vs SM } \\
\hline Especie & $\mathrm{Pd}$ & $\%$ Contr. & $\%$ Contr. A \\
\hline Thouinia paucidentata Radlk. & 20.06 & 22.31 & 22.31 \\
\hline Piscidia piscipula (L.) Sarg. & 10.04 & 11.17 & 33.48 \\
\hline Lysiloma latisiliquum (L.) Benth. & 6.857 & 7.626 & 41.1 \\
\hline Lonchocarpus castilloi Standl. & 5.08 & 5.65 & 46.75 \\
\hline Vitex gaumeri Greenm. & 4.292 & 4.773 & 51.52 \\
\hline Cedrela odorata L. & 3.797 & 4.223 & 55.75 \\
\hline Spondias mombin L. & 3.625 & 4.032 & 59.78 \\
\hline Astronium graveolens Jacq. & 3.535 & 3.932 & 63.71 \\
\hline Bursera simaruba (L.) Sarg. & 3.044 & 3.385 & 67.1 \\
\hline
\end{tabular}

Pd = Promedio de disimilitud; \% Contr. = Porcentaje de contribución; $\%$ Contr. A = Porcentaje de contribución acumulado. 
En el análisis de la estructura, el área basal promedio del A fue de $39.08 \mathrm{~m}^{2} \pm 0.71$, la del JB de $14.12 \mathrm{~m}^{2} \pm 2.98$ y la de las SM de $38.93 \mathrm{~m}^{2} \pm 9.37$. Las especies con mayor VIR para cada una de las áreas se muestran en el Cuadro 3. Se observa que en el JB y el A el valor de VIR está asociado con cifras altas de abundancia relativa o del área basal relativa; mientras que, en las SM hay especies en las que la frecuencia relativa es responsable del alto valor de VIR.

Cuadro 3. Lista de especies con mayor valor de importancia relativa (VIR) en el arboretum (A), jardín botánico (JB) y selvas (SM) de El Tormento.

\begin{tabular}{|c|c|c|c|c|}
\hline Especie & Frec rel & Ab rel & $A B$ rel & VIR \\
\hline \multicolumn{5}{|l|}{ A } \\
\hline Lysiloma latisiliquum (L.) Benth. & 0.00 & 11.84 & 21.31 & 11.05 \\
\hline Piscidia piscipula (L.) Sarg. & 2.67 & 15.66 & 11.30 & 9.88 \\
\hline Enterolobium cyclocarpum (Jacq.) Griseb. & 2.67 & 3.01 & 14.99 & 6.89 \\
\hline Lonchocarpus castilloi Standl. & 2.67 & 8.01 & 9.60 & 6.76 \\
\hline Vitex gaumeri Greenm. & 2.67 & 7.01 & 8.60 & 6.09 \\
\hline Spondias mombin L. & 2.67 & 5.74 & 4.54 & 4.31 \\
\hline Bursera simaruba (L.) Sarg. & 2.67 & 5.46 & 4.59 & 4.24 \\
\hline Cedrela odorata L. & 2.67 & 6.10 & 3.88 & 4.22 \\
\hline Astronium graveolens Jacq. & 2.67 & 5.92 & 2.59 & 3.72 \\
\hline \multicolumn{5}{|l|}{$\mathrm{JB}$} \\
\hline Lysiloma latisiliquum (L.) Benth. & 2.08 & 1.65 & 14.43 & 6.05 \\
\hline Cryosophila stauracantha (Heynh.) R.J.Evans & 2.08 & 10.68 & 1.83 & 4.86 \\
\hline Pouteria reticulata (Engl.) Eyma & 2.08 & 8.83 & 3.48 & 4.79 \\
\hline Nectandra salicifolia (Kunth) Nees. & 2.08 & 10.33 & 1.86 & 4.75 \\
\hline
\end{tabular}




\begin{tabular}{lllll} 
Metopium brownei (Jacq.) Urb. & 2.08 & 6.27 & 5.50 & 4.62 \\
Alseis yucatanensis Standl. & 2.08 & 4.47 & 4.31 & 3.62 \\
Bursera simaruba (L.) Sarg. & 2.08 & 2.30 & 5.28 & 3.22 \\
Vitex gaumeri Greenm. & 2.08 & 1.12 & 6.11 & 3.10 \\
Manilkara zapota L(L.) P.Royen & 2.08 & 1.52 & 5.53 & 3.04 \\
\hline SM & & & & \\
\hline Piscidia piscipula (L.) Sarg. & 15.09 & 2.78 & 12.67 & 10.18 \\
Pimenta dioica (L.) Merrill & 2.26 & 0.93 & 19.12 & 7.44 \\
Lysiloma latisiliquum (L.) Benth. & 2.64 & 3.70 & 9.85 & 5.40 \\
Vitex gaumeri Greenm. & 2.45 & 1.85 & 9.81 & 4.70 \\
Guettarda combsii Urb & 7.55 & 3.70 & 1.63 & 4.29 \\
Bursera simaruba (L.) Sarg. & 3.40 & 3.70 & 3.97 & 3.69 \\
Metopium brownei (Jacq.) Urb. & 2.83 & 3.70 & 2.94 & 3.16 \\
Lonchocarpus castilloi Standl. & 3.21 & 2.78 & 3.40 & 3.13 \\
Blepharidium guatemalense Standl. & & 2.78 & 2.61 & 3.05 \\
\hline
\end{tabular}

Las estructuras vertical y horizontal en el JB se caracterizaron por una distribución en forma de "J invertida", con más de $70 \%$ de individuos en las dos primeras categorías. En A y en las SM la distribución es en forma de campana, en la que las categorías intermedias ( 2 a 4 ) concentran alrededor de $75 \%$ de los individuos (figuras 2 y 3 ). 


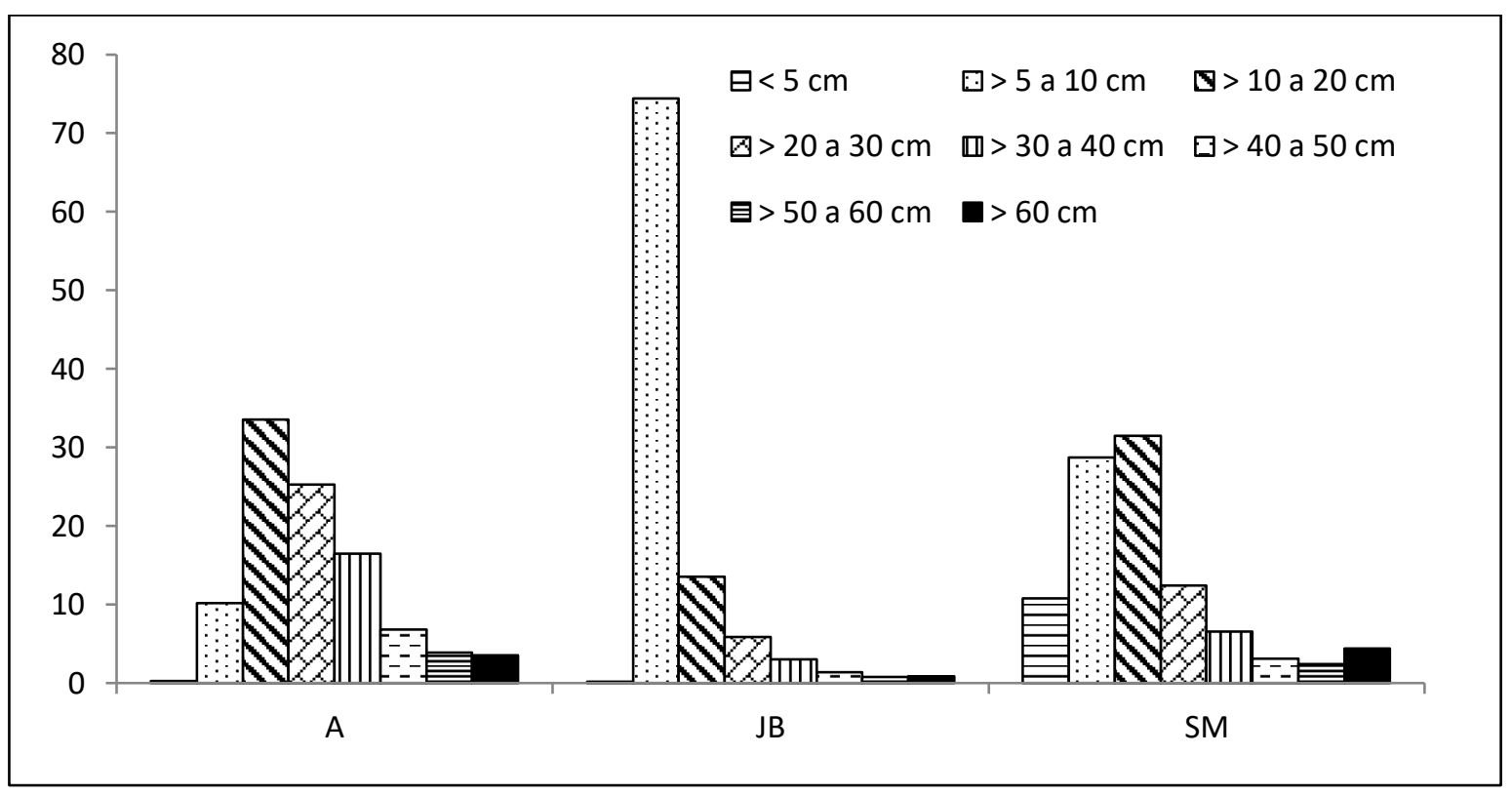

Figura 2. Estructura horizontal (diámetros) en el arboretum (A), jardín botánico (JB) y selvas (SM) de El Tormento.

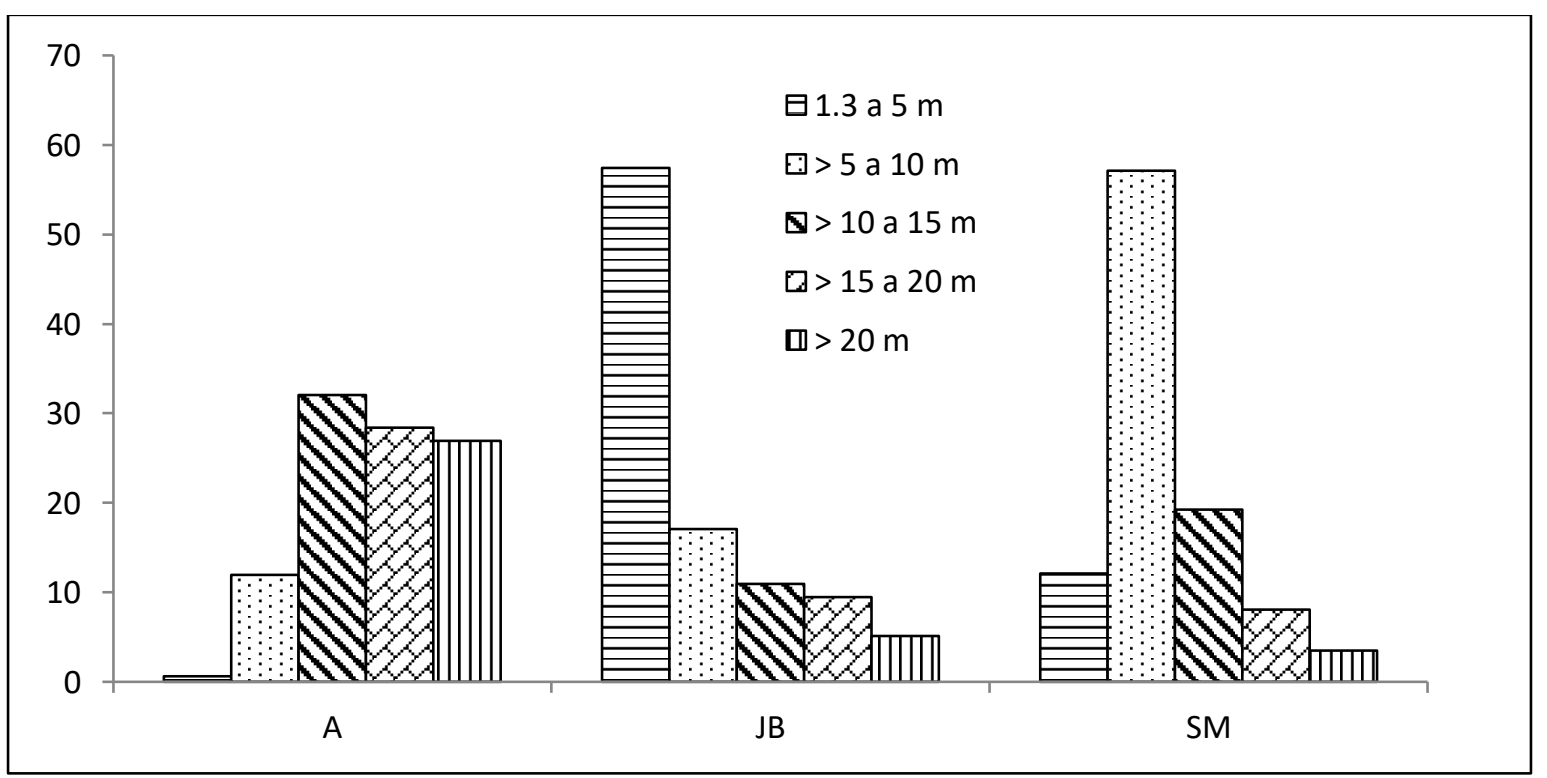

Figura 3. Estructura vertical (altura) en las áreas del arboretum (A), jardín botánico (JB) y selvas (SM) de El Tormento. 
El número de especies efectivas evidenció que el JB $(1 \mathrm{D}=32.82)$ es 1.6 veces más diverso que el $A(1 D=20.8)$. En cuanto a la completitud del inventario, ambos sitios tuvieron valores altos; en el JB los números estimados se distribuyeron entre $80.69 \%$ (Chao 2: 109.05), $92.09 \%$ (ACE: 95.56) y $94.48 \%$ (Chao 1: 93.14); mientras que para el A las estimaciones variaron de $79.27 \%$ (ACE: 64.33), $88.81 \%$ (Chao 1: 57.42 ) a $92.12 \%$ (Chao 2: 55.36). Todos los estimadores predijeron una mayor riqueza en el JB (cinco a 21 nuevas especies), respecto al A (cuatro a 13 nuevas especies).

\section{Discusión}

El número de especies y familias registradas en este estudio para el JB y el A, concuerda con las 10 familias más frecuentes y con mayor número de especies reconocidas para la Península de Yucatán (Carnevali et al., 2010); y, parcialmente. con los resultados del trabajo de Salaya-Sánchez y Gómez-Gómez (1981), quienes registraron que en el JB de El Tormento las familias mejor representadas, en cuanto a especies, eran Fabaceae (14), Euphorbiaceae (6), Rubiaceae, Moraceae y Sapotaceae (5). Mientras que, las más abundantes eran Rubiaceae (839), Fabaceae (582), Lauraceae (500) y Anacardiaceae (437). Todas las especies en ambas áreas se han recolectado en selvas medianas subperennifolias y algunas de ellas, como Pouteria reticulata, se consideran características de selvas maduras; otras como Nectandra salicifolia pueden presentarse desde las etapas tempranas de la sucesión.

Los resultados indican la importancia del JB y del A como reservorios de especies forestales representativas de selvas medianas subperennifolias, útiles desde muy diversas perspectivas y como espacios de conservación de especies amenazadas. Lo anterior coincide con lo consignado por Chen et al. (2009), Oldfield (2009), Miller et al. (2016), Gaio-Oliveira et al. (2017), Volis (2017), así como Chen y Sun (2018) quienes destacan el valor de estos espacios como centros de diversidad, diseño de estrategias de conservación, manejo e investigación de los recursos forestales, además para salvaguardar la biodiversidad asociada a los diferentes ecosistemas representados. 
En los dos sitios sobresale el alto porcentaje de especies con múltiples usos, lo que evidencia la enorme riqueza de componentes bióticos y, en consecuencia, de servicios ambientales que ofrecen los taxa arbóreos de las selvas. En este contexto, se pueden mencionar: el abastecimiento de madera, medicinas, alimentos, forraje y cercos vivos; pero también, la presencia de especies melíferas que favorecen la polinización y la de aquellas con un alto valor cultural y religioso. Al respecto, Hardwick et al. (2011) enfatizan el papel de los jardines botánicos como custodios de taxa importantes para las comunidades locales por los servicios ecosistémicos que proporcionan.

Chen et al. (2009) subrayan la trascendencia de documentar los usos de especies nativas, desarrollar estrategias de manejo en los jardines botánicos tropicales y difundir ambos entre los diferentes usuarios del bosque, para favorecer su conservación. Otros autores se enfocan en las especies amenazadas o endémicas con utilidad y relevancia para atender las necesidades de las comunidades, en relación con salud, nutrición, silvicultura, combustibles y agricultura, particularmente en el contexto del cambio climático (Hardwick et al., 2011; Heywood, 2011; Chen y Sun, 2018). Cannon y Kua (2017), así como Dunn (2017) destacan la necesidad de incorporar perspectivas socioeconómicas en los planes de conservación de los jardines botánicos, que integren los usos locales, tradicionales y el valor cultural, es decir con un contexto biocultural.

Tanto el arboretum como el jardín botánico cumplen con la función de resguardar especies con algún grado de amenaza, asimismo constituyen reservorios y fuentes potenciales de germoplasma para la conservación de las especies. En ese sentido, Oldfield (2009) resalta la importancia que tienen los jardines botánicos en la conservación de especies arbóreas y enfatiza la necesidad de espacios que representen el hábitat natural. Chen et al. (2009) señalan la relevancia de tener jardines botánicos tropicales in-situ, que promuevan iniciativas, no solo para la conservación de taxones arbóreos nativos y su reintroducción, sino también por la gran biodiversidad que pueden albergar y conservar. Otros autores (Heywood, 2017; Volis, 2017) documentan la importancia de estos espacios para priorizar la 
conservación de especies a nivel regional, a partir de su vulnerabilidad a los efectos del cambio climático y la posibilidad de comprobar sus impactos en los procesos de floración, hibridación, polinización, colonización de especies invasoras o bien en la plasticidad que algunas de ellas pueden mostrar.

Por otro lado, Miller et al. (2016) consideraron que las colecciones vivas aquí estudiadas tienen una gran repercusión en la generación y el monitoreo de estrategias de restauración, puesto que son áreas que reúnen información relacionada con la sucesión, la composición de especies de ecosistemas de referencia, la correcta propagación de las especies forestales; a la par que son espacios que contribuyen a divulgar la importancia de cuidar y hacer un manejo sustentable de los recursos forestales.

A pesar del alto valor en el porcentaje de disimilitud entre la composición de las SM $v s$ el JB y el A, es innegable la conveniencia de que el JB alberge más especies de las presentes en las SM, particularmente, aquellas con algún estatus de amenaza o y un alto potencial de uso.

Los valores del $A B$ de las $S M$ y el $A$ son semejantes a los consignados por diversos autores para selvas medianas subperennifolias del sur de México, como Reed y Lawrence (2003), García-Licona et al. (2014), Esparza-Olguín y Martínez-Romero (2018); mientras que en JB, las $A B$ son comparables con las de vegetación secundaria (Reed y Lawrence, 2003; Van Breugel et al., 2006; Vester et al., 2007; García-Licona et al. 2014; Esparza-Olguín et al., 2019). En el JB y en el A sobresalen especies registradas para las selvas medianas subperennifolias $y$ vegetación secundaria derivada de estas con altos valores de VIR (Díaz et al., 2002; Read y Lawrence, 2003; Pennington y Sarukhán, 2005; Vester et al., 2007; Zamora-Crescencio et al., 2012; García-Licona et al., 2014; Esparza-Olguín y Martínez-Romero, 2018).

En relación con el comportamiento de las estructuras horizontal y vertical, tanto el JB (J invertida) como el A y las SM (en forma de campana) coinciden con el argumento de que ambos patrones indican que las áreas mantienen procesos de 
recuperación a través de la sucesión, y así garantizan el reemplazo de árboles eliminados por diversas causas (Díaz et al., 2002; Zamora-Crescencio et al., 2012; García-Licona et al., 2014; Báez-Vargas et al., 2017; Chiquini-Heredia et al., 2017). Las diferencias en la diversidad entre el JB y el A, probablemente, estén asociadas con el hecho de que el JB es un espacio que no recibió manejo o mantenimiento por más de 35 años, por lo que su vegetación siguió el proceso de sucesión ecológica, con el consecuente recambio de especies, lo que originó una mayor diversidad (Poorter, 2007; Norden et al., 2009; Chazdon et al., 2010; Lebrija et al., 2010). En el $A$ se hicieron acciones esporádicas de mantenimiento que contribuyeron a que se conservara como una colección de árboles vivos con el arreglo de una plantación.

Los estimadores de riqueza de especies utilizados señalan un esfuerzo de muestro suficiente que permite una buena representación de la composición de especies tanto para el JB, como para el A, dados los valores relativamente altos de completitud (Álvarez et al., 2012; Vite et al., 2014).

\section{Conclusiones}

Los resultados del presente estudio en su conjunto confirman la importancia del Jardín Botánico y el Arboretum como reservorios de la diversidad arbórea asociada a las selvas medianas subperennifolias y su uso potencial; constituyen espacios para la formulación de estrategias de conservación, manejo y conocimiento de los recursos forestales. Al formar parte de un centro de investigación y transferencia de tecnología, la información vertida en este trabajo coadyuvará en la generación y desarrollo de líneas de investigación y propagación de las especies arbóreas y de la biodiversidad asociada a las selvas a nivel local y regional. Así mismo, los datos aquí reunidos podrán fundamentar el diseño de planes de educación y divulgación vinculados a las diferentes temáticas que se trabajen en ambas colecciones vivas. 


\section{Agradecimientos}

Los autores agradecen a la Comisión Nacional Forestal (Conafor) por el financiamiento del proyecto "Reactivación de las Investigaciones forestales en el Centro de Investigación y Transferencia de Tecnología Forestal El Tormento". A los parataxónomos Manuel Arana Cua, Demetrio Montejo Álvarez y Antonio López Carrillo por su apoyo en la determinación de especies. A la dirección y personal del Centro de Investigación y Transferencia de Tecnología Forestal "El Tormento" por las facilidades otorgadas para la realización de este trabajo.

\section{Conflicto de intereses}

Los autores declaran no tener conflicto de intereses.

\section{Contribución por autor}

Ligia Guadalupe Esparza Olguín: análisis de datos, elaboración del manuscrito en todos sus apartados; Aixchel Maya Martínez: trabajo de campo, elaboración de diferentes secciones del manuscrito y revisión general del artículo; Gonzalo Hernández García: trabajo de campo, revisión general del artículo; Eduardo Martínez Romero: elaboración de diferentes secciones del manuscrito y revisión general del artículo.

\section{Referencias}

Álvarez Z., E., A. Sánchez G., L. López M. y J. D. Tejero D. 2012. Composición y abundancia de las pteridofitas en el bosque mesófilo de montaña del municipio de Tlanchinol, Hidalgo, México. Botanical Sciences 90: 163-177. Doi: 10.17129/botsci.481. 
Arnet, M., B. Santos, E. G. Brocherhoff, P. B. Pelser, C. Ecroyd and J. Clemens. 2015. Importance of arboreta for ex situ conservation of threatened trees. Biodiversity and Conservation 24: 3601-3620. Doi: 10.1007/s10531-015-1024-3.

Báez-Vargas, A. M., L. Esparza O., E. Martínez R., S. Ochoa G., N. Ramírez-M. y N. A. González V. 2017. Efecto del manejo sobre la diversidad de árboles en vegetación secundaria en la Reserva de la Biosfera de Calakmul, Campeche, México. Revista de Biología Tropical 65(1): 41-53. Doi: 10.15517/RBT.V65I1.20806.

Balvanera, P. 2012. Los servicios ecosistémicos que ofrecen los bosques tropicales. Ecosistemas 21: 136-147.

Bray, J. R. and J. T. Curtis. 1957. An ordination of the upland forest communities of Southern Wisconsin. Ecological Monographs 27: 326-349.

Cannon C. H. and C. S. Kua. 2017. Botanic gardens should lead the way to create a "Garden Earth" in the Anthropocene. Plant Diversity 39: 331-337. Doi: 10.1016/j.pld.2017.11.003

Carnevali, F., C., G. J. L. Tapia-Muñoz, R. Duno de Stefano y I. Ramírez-Morillo. 2010. Flora ilustrada de la Península de Yucatán: listado florístico. Centro de Investigación Científica de Yucatán, A. C. Mérida, Yuc., México. 326 p.

Cedeño, O. 1981. Campo Experimental Forestal "El Tormento" Campeche. Revista Ciencia Forestal 1 (3): 75-82.

Challenger, A. y J. Soberón. 2008. Los ecosistemas terrestres. In: Soberón J., G. Halffter y J. Llorente B. (comps.). Capital natural de México. Vol I: Conocimiento Actual de la Biodiversidad México. Comisión Nacional para el Conocimiento y Uso de la Biodiversidad. México D. F., México. pp. 87-108. 
Chazdon, R. L., B., B. Finegan, R. S. Capers, B. Salgado N., F. Casanoves, V. Boukili and N. Norden. 2010. Composition and dynamics of functional groups of tres during tropical forest succession in Northeastern Costa Rica. Biotropica 42 (1): 31-40. Doi: 10.1111/j.1744-7429.2009.00566.x.

Chen, J., C. H. Cannon and H. Hu. 2009. Tropical botanical gardens: at the in situ ecosystem management frontier. Trends in Plant Science 14 (11): 584-589. Doi: $10.1016 /$ j.tplants.2009.08.010.

Chen, G. and W. Sun. 2018. The role of botanical gardens in scientific research, conservation, and citizen science. Plant Diversity 40: 181-188. Doi: 10.1016/j.pld.2018.07.006.

Chiquini-Heredia W., L. Esparza O., Y. Peña R., A. Maya M. y E. Martínez R. 2017. Estructura y diversidad en selva inundable al centro y sur de Calakmul. Ecosistemas y Recursos Agropecuarios 4: 511-524. Doi: 0.19136/era.a4n12.859.

Convención sobre el Comercio Internacional de Especies (CITES). 2017. Apéndices I, II y III. https://www.cites.org/esp/app/appendices.php (12 de noviembre de 2019).

Clarke, K. R. 1993. Non-parametric multivariate analyses of changes in community structure. Australian Journal of Ecology 18: 117-143. Doi: 10.1111/j.1442-9993.1993.tb00438.x.

Colwell, R. K. 2017. EstimateS9.1: Statistical estimation of species richnes and shared species from samples. http://viceroy.eeb.uconn.edu/estimates/index.html (10 de abril de 2019).

Cuanalo, C. H. E. 1981. Suelos del Campo Experimental Forestal "El Tormento". Boletín Técnico 15. INIF. Campeche, Camp., México. 32 p.

Díaz G., J. R., O. Castillo A. y G. García G. 2002. Distribución espacial y estructura arbórea de la selva baja subperennifolia en un ejido de la Reserva de la Biosfera de Calakmul, Campeche, México. Universidad y Ciencia 18: 11-28. http://ri.ujat.mx/bitstream/20.500.12107/1389/1/-235-191-A.pdf (10 de abril de 2019). 
Dirzo, R., A. Aguirre y J. C. López. 2009. Diversidad florística de las selvas húmedas en paisajes antropizados. Investigación Ambiental 1(1): 17-22.

Dunn, C. P. 2017. Biological and cultural diversity in the context of botanic garden conservation strategies. Plant Diversity 39: 396-401. Doi: 10.1016/j.pld.2017.10.003.

Food and Agriculture Organization of the United Nations (FAO). 2016. El estado de los bosques del mundo 2016. Los bosques y la agricultura: desafíos y oportunidades en relación con el uso de la tierra. Organización de las Naciones Unidas para la Agricultura y Alimentación. Roma, Italia. 137 p.

Esparza-Olguín, L. y E. Martínez-Romero. 2018. Diversidad y carbono almacenado en el área forestal permanente de Álvaro Obregón, Campeche. Revista Mexicana de Ciencias Forestales 9: 152-186. Doi: 10.29298/rmcf.v9i45.141.

Esparza-Olguín, L., J. A. Vargas C., E. Martínez R. y G. Escalona S. 2019. Diversidad y biomasa de la selva circundante al Volcán de los Murciélagos, en Campeche, México. Ecosistemas y Recursos Agropecuarios 6: 79-90. Doi: 10.19136/era.a6n16.1986.

Gaio-Oliveira, G., A. Delicado and M. A. Martins L. 2017. Botanic gardens as communicators of plant diversity and conservation. The Botanical Review 83: 282302. Doi: $10.1007 / \mathrm{s} 12229-017-9186-1$.

García-Licona, J. B., L. Esparza O. y E. Martínez R. 2014. Estructura y composición de la vegetación leñosa de selvas en diferentes estadios sucesionales en el ejido El Carmen II, Calakmul, México. Polibotánica 38: 1-26.

https://www.encb.ipn.mx/assets/files/encb/docs/polibotanica/revistas/pb38/calak.pdf (12 de mayo de 2019).

Unión Internacional para la Conservación de la Naturaleza (IUCN). 2019. The IUCN Red List of Threatened Species. Version 2018-2. http://www.iucnredlist.org (4 de diciembre 2019). 
Hammer, Ø., D. A. T. Harper and P. D. Ryan. 2001. PAST 3.25: Paleontological Statistics Software Package for Education and Data Analysis. http://nhm2.uio.no/norlex/past/download.html (10 de abril de 2019).

Hardwick, K. A., P. Fiedler, L. Lee, B. Pavlik, R. J. Hobbs, J. Aronson, M. Bidartondo, E. Black, D. Coates, M. I. Daws, K. Dixon, S. Elliott, K. Ewing, G. Gann, D. Gibbons, J. Gratzfeld, M. Hamilton, D. Hardman, J. Harris, P. M. Holmes, M. Jones, D. Mabberley, A. Mackenzie, C. Magdalena, R. Marrs, W. Milliken, A. Mills, E. N. Lughadha, M. Ramsay, P. Smith, N. Taylor, C. Trivedi, M. Way, O. Whaley and S. D. Hopper. 2011. The role of botanic gardens in the science and practice of ecological restoration. Conservation Biology 25: 265-275.

Doi: $10.1111 / \mathrm{j} .1523-1739.2010 .01632 . x$.

Heywood, V. H. 2011. The role of botanic gardens as resource and introduction centres in the face of global change. Biodiversity Conservation 20: 221-239. Doi: $10.1007 / \mathrm{s} 10531-010-9781-5$.

Heywood, V. H. 2017. The future of plant conservation and the role of botanic gardens. Plant Diversity 39(6): 309-313. Doi: 10.1016/j.pld.2017.12.002.

Jost, L. y J. A. González-Oreja. 2012. Midiendo la diversidad biológica: más allá del índice de Shannon. Acta Zoológica Lilloana 56: 3-14.

Lawrence, D., H. F. M. Vester, D. Pérez S., J. R. Eastman, B. L. Turner II and J. Geoghegan. 2004. Integrated analysis of ecosystem interactions with land-usechange: The Southern Yucatán peninsular region. In: Defries, R. S., G. P. Asner and R. A. Houghton (eds.). Ecosystems and land use change. American Geophysical Union. Washington D.C., USA. pp. 277-292.

Lebrija T., E., J. A. Meave, L. Poorter, E. A. Pérez G. and F. Bongers. 2010. Pathways, mechanisms and predictability of vegetation change during tropical dry forest succession. Perspectives in Plant Ecology, Evolution and Systematics 12: 267275. Doi: $10.1016 /$ j.ppees.2010.09.002. 
López-Torres, J. L. y J. C. Tamarit-Urías. 2005. Crecimiento e incremento en diámetro de Lysiloma latisiliquum (L.) Benth. en bosques secundarios en Escárcega, Campeche, México. Revista Chapingo. Serie Ciencias Forestales y del Ambiente 11(2): 117-123.

Magurran, A. E. 2004. Measuring biological diversity. Blackwell Science. Oxford, UK, 261 p. Miller, J. S., P. Porter, J. A. Lowry II, S. Blackmore, K. Havens and J. Maschinski. 2016. Conservating biodiversity through Ecological Restoration: the potential contributions of botanical gardens and arboreta. Candollea 71 (1): 91-98. Doi: $10.15553 / c 2016 v 711 a 11$.

Norden, N., R. L. Chazdon, A. Chao, Y. H. Jiang, and B. Vilchez A. 2009. Resilience of tropical rain forests: Tree community reassembly in secondary forests. Ecology Letters 12: 395-394. Doi: 10.1111/j.1461-0248.2009.01292.x.

Oldfield, S. F. 2009. Botanic gardens and the conservation of tree species. Trends in Plant Science 14 (11): 581-583. Doi: 10.1016/j.tplants.2009.08.013.

Pennington, T. D. y J. Sarukhán. 2005. Árboles tropicales de México. Manual para la identificación de las principales especies. Universidad Nacional Autónoma de México y Fondo Cultura Económica. México, D.F., México. 523 p.

Pérez-Sarabia, J. E., R. de Stefano, G. Carnevali F. C., I. Ramírez M.o, N. Méndez J., P. Zamora C., C. Gutiérrez B. y W. Cetzal I. 2017. El conocimiento florístico de la Península de Yucatán, México. Polibotánica 44: 39-49. Doi: 10.18387/polibotanica.44.3.

Poorter, L. 2007. Are species adapted to their regeneration niche, adult niche, or both? American Naturalist 169: 433-442. Doi: 10.1086/512045.

Reed, L. and D. Lawrence. 2003. Recovery of biomass following shifting cultivation in dry tropical forest of the Yucatan. Ecological Applications 13(1): 85-97.

Doi: 10.1890/1051-0761(2003)013[0085:ROBFSC]2.0.CO;2. 
Salaya-Sánchez, A. y G. Gómez-Gómez. 1981. Consideraciones sobre un jardín natural forestal. Revista Ciencia Forestal 33(6): 43-64.

Secretaría de Medio Ambiente y Recursos Naturales (Semarnat). 2010. Norma Oficial Mexicana NOM-059-SEMARNAT-2010, Protección ambiental - Especies nativas de México de flora y fauna silvestres - Categorías de riesgo y especificaciones para su inclusión, exclusión o cambio - Lista de especies en riesgo. México. Diario Oficial de la Federación. Secretaría de Gobernación. México, D.F., México. http://www.dof.gob.mx/normasOficiales/4254/semarnat/semarnat.htm (21 de enero de 2019).

Sloan, S. and J. A. Sayer. 2015. Forest Resources Assessment of 2015 shows positive global trends but forest loss and degradation persist in poor tropical countries. Forest Ecology and Management 352: 134-145. Doi: 10.1016/j.foreco.2015.06.013

The Plant List. 2013. The Plant list. Ver. 1.1 http://www.theplantlist.org/ (21 de enero de 2019).

Uzcanga-Pérez, N. G., Y. Aguilar, A. Maya, M. Díaz y L. Esparza O. 2018. Compendio histórico de la investigación realizada en el Centro de Investigación y Transferencia de Tecnología Forestal El Tormento. Publicación Especial Núm. 10. Instituto Nacional de Investigaciones Forestales, Agrícolas y Pecuarias. Campo Experimental Mocochá. Centro de Investigación Regional Sureste. Mocochá, Yuc., México. 36 p.

Van Breugel, M., M. Martínez R. and F. Bongers. 2006. Community dynamics during early secondary succession in Mexican tropical rain forest. Journal of Tropical Ecology 22: 663-674. Doi: 10.1017/S0266467406003452.

Vester, H. F. M., D. Lawrence, J. R. Eastman, B. L. Turner, S. Calmé, R. Dickson, C. Pozo and F. Sangermano. 2007. Land change in the Southern Yucatán and Calakmul Biosphere Reserve: Effects on habitat and Biodiversity. Ecological Applications 17: 989-995. Doi: 10.1890/05-1106. 
Vite C., C., J. L. Alanís M., J. M. Pech C. y E. Ramos H. 2014. Indicadores de diversidad, estructura y riqueza para la conservación de la biodiversidad vegetal en los paisajes rurales. Tropical and Subtropical Agroecosystems 17: 185-196.

Volis, S. 2017. Conservation utility of botanic garden living collections: Setting a strategy and appropriate methodology. Plant Diversity 39(6): 365-372.

Doi: $10.1016 / j . p l d .2017 .11 .006$

Zamora-Crescencio, P., C. Gutiérrez-Báez, W. J. Folan, M. R. Domínguez-Carrasco, P. Villegas, G. Cabrera-Mis, C. M. Castro-Angulo y J. C. Carballo. 2012. La vegetación leñosa del sitio arqueológico de Oxpemul, municipio de Calakmul, Campeche, México. Polibotánica 33: 131-150. distribuyen amparados bajo la licencia Creative Commons 4.0 Atribución-No Comercial (CC BY-NC 4.0 Internacional), que permite a terceros utilizar lo publicado siempre que mencionen la autoría del trabajo y a la primera publicación en esta revista. 